\title{
Absolute risk of tuberculosis among untreated populations with a positive tuberculin skin test or interferon-gamma release assay result: systematic review and meta-analysis
}

\author{
Jonathon R Campbell, ${ }^{1,2}$ Nicholas Winters, ${ }^{1,2}$ Dick Menzies ${ }^{1,2,3}$
}

${ }^{1}$ Department of Epidemiology,

Biostatistics, and Occupational Health, McGill University,

Montreal, QC, Canada

${ }^{2}$ McGill International TB Centre, Montreal, QC, Canada

${ }^{3}$ Montreal Chest Institute,

Montreal, QC, Canada

Correspondence to: D Menzies, McGill University, Office 3D.58,

5252 Boulevard de Maisonneuve O,

Montreal, QC, H4A 3S5, Canada

dick.menzies@mcgill.ca

(ORCID 0000-0003-1601-4514)

Additional material is published online only. To view please visit the journal online.

Cite this as: BMJ 2020;368:m549 http://dx.doi.org/10.1136/bmj.m549

Accepted: 22 January 2020

\section{ABSTRACT}

OBJECTIVE

To determine the annual rate of tuberculosis development after a positive tuberculin skin test (TST) or interferon-gamma release assay result (IGRA), or both, among untreated populations with characteristics believed to increase the risk of tuberculosis (at risk populations).

DESIGN

Systematic review and meta-analysis.

DATA SOURCES

Embase, Medline, and Cochrane Controlled Register of Trials from 1 January 1990 to 17 May 2019, for studies in humans published in English or French. Reference lists were reviewed.

\section{ELIGIBILITY CRITERIA AND DATA ANALYSIS}

Retrospective or prospective cohorts and randomised trials that included at least 10 untreated participants who tested positive to tuberculosis antigens (contained in TST or IGRA, or both) followed for at least 12 months. Following the preferred reporting items for systematic reviews and meta-analyses (PRISMA) and meta-analyses of observational studies in epidemiology (MOOSE) guidelines, two reviewers independently extracted study data and assessed quality using a modified quality assessment of diagnostic accuracy studies (QUADAS-2) tool. Data were pooled using random effects generalised linear mixed models.

MAIN OUTCOME MEASURES

The primary outcome was tuberculosis incidence per 1000 person years among untreated participants

\section{WHAT IS ALREADY KNOWN ON THIS TOPIC}

In the testing for and treatment of latent tuberculosis (TB) infection, international TB guidelines prioritise populations with recent exposure to TB or with concomitant health risks that affect immunocompetency and risk of exposure to TB

Many of the risk estimates that inform these guidelines are based on older literature and presented as relative risks, which conflate two different risksacquiring or having latent TB infection and progression to active disease Therefore, among people with a positive test result for latent TB infection, absolute risk of progression to active disease is unclear

\section{WHAT THIS STUDY ADDS}

Rates of progression to active TB were highest among TB contacts, people living with HIV, recent immigrants or refugees, people requiring dialysis, people with silicosis, and prisoners

Incidence rate ratios suggest diagnostic tests for latent TB infection are useful in discriminating between people at higher and lower risk of TB among immunocompetent people and those living with HIV who tested positive (TST or IGRA, or both) in different at risk subgroups. Secondary outcomes were the cumulative incidence of tuberculosis and incidence rate ratios among participants with a positive test result for latent tuberculosis infection compared with those with a negative test result in at risk subgroups.

\section{RESULTS}

122 of 5166 identified studies were included. In three general population studies, the incidence of tuberculosis among 33811 participants with a TST induration of $\geq 10 \mathrm{~mm}$ was 0.3 (95\% confidence interval 0.1 to 1.1$)$ per 1000 person years. Among 116197 positive test results for latent tuberculosis infection in 19 different at risk populations, incidence rates were consistently higher than those in the general population. Among all types of tuberculosis contacts, the incidence of tuberculosis was 17.0 (95\% confidence interval 12.9 to 22.4 ) per 1000 person years for participants with a positive IGRA result and 8.4 (5.6 to 12.6 ) per 1000 person years for participants with a positive TST result of $\geq 5 \mathrm{~mm}$. Among people living with HIV, the incidence of tuberculosis was 16.9 (10.5 to 27.3) for participants with a positive IGRA result and 27.1 (15.0 to 49.0 ) for participants with a positive TST result of $\geq 5 \mathrm{~mm}$. Rates were also high for immigrants, people with silicosis or requiring dialysis, transplant recipients, and prisoners. Incidence rate ratios among test positive versus test negative participants were significantly greater than 1.0 in almost all risk groups, for all tests.

\section{CONCLUSIONS}

The incidence of tuberculosis is substantial in numerous at risk populations after a positive TST or IGRA result. The information from this review should help inform clinical decisions to test and treat for latent tuberculosis infection.

STUDY REGISTRATION

PROSPERO CRD42019136608.

\section{Introduction}

Testing people for latent tuberculosis infection and providing effective treatment is critical to elimination of the disease. ${ }^{1}$ Deciding to test for latent infection depends on both the probability of infection and the risk for progression to active disease. ${ }^{2}$ As part of the assessment for latent tuberculosis infection, the tuberculin skin test (TST) or commercially available interferon-gamma release assay (IGRA; T-SPOT.TB or QuantiFERON-TB (QFT)), or both, are commonly used. ${ }^{3}$ The TST and IGRA rely on an adaptive immune response to tuberculosis antigens. The TST involves an injection of non-specific tuberculosis antigens, which induces a delayed type hypersensitivity reaction that can be 
measured in infected individuals, whereas IGRAs are blood tests that measure interferon $\gamma$ produced by lymphocytes in whole blood in the presence of tuberculosis specific antigens. ${ }^{4}$ In the presence of a positive test result for latent infection, treatment depends on a careful consideration of the benefits and risks. ${ }^{5}$ These primarily include the benefit of reducing an individual's probability of developing tuberculosis and the risk of treatment related side effects, which are substantial with current treatment regimens for latent tuberculosis infection. ${ }^{6}$

Only a few people with a positive test result for latent tuberculosis infection will progress to active disease. $^{7}$ The risk for progression is affected by time since infection and integrity of the individual's immune system, which might be compromised as a result of drugs, habits (intravenous drug use, smoking, and alcohol use), or underlying conditions such as HIV infection. ${ }^{2}$ Although populations with any of these characteristics (at risk populations) are generally prioritised for tuberculosis testing and treatment, ${ }^{28-11}$ the exact risks for progression are not well established. ${ }^{12}$ With changing global tuberculosis epidemiology, including the introduction of effective treatment for HIV, better living conditions, and longer life expectancies for immunocompromised people, updated quantification of these risks in the modern era are urgently needed.

Previous summary estimates of tuberculosis incidence in at risk populations have been benchmarked against the general population but have not compared populations on the basis of diagnostic test results for latent tuberculosis infection. The estimates are thus a composite risk of the probability that someone has or will acquire latent tuberculosis infection and the probability someone will progress to active disease. ${ }^{2} 9$ This conflates two different phenomena, which are influenced by different factors: risk of latent tuberculosis infection is determined by local epidemiology, transmission dynamics, and patient history, ${ }^{13}{ }^{14}$ whereas the risk of progression is determined by time since infection and immunocompetency. ${ }^{2}$ To help inform decision making by providers and patients, ${ }^{15}$ we conducted a systematic review and meta-analysis to determine the absolute risk of development of active tuberculosis among different populations of untreated people with a positive TST or IGRA result, or both.

\section{Methods}

In this meta-analysis we followed the preferred reporting items for systematic reviews and metaanalyses (PRISMA) and meta-analyses of observational studies in epidemiology (MOOSE) guidelines. ${ }^{16} 17$

\section{Search strategy and selection criteria}

Because an earlier review ${ }^{12}$ including studies from 1949 to 2003 examining risk for progression to active tuberculosis disease indicated that older studies might be confounded by previously healed tuberculosis ${ }^{18}$ or by high levels of ongoing transmission, we limited our search to studies indexed from 1 January 1990 to 17 May 2019 in Medline, Embase, or the Cochrane Controlled Register of Trials. We restricted the search to studies conducted in humans and published in English or French (see supplementary tables S1 to S3 for search strategies). Two reviewers (JRC and NW) independently reviewed titles, abstracts, and full texts and resolved disagreements with a third reviewer (DM). The reference lists of selected full texts and identified systematic reviews were reviewed for additional studies.

We included clinical trials and retrospective or prospective cohort studies in which more than 50\% of participants were recruited from 1990 onwards. Included studies reported TST results at internationally accepted cut points for indurations ( $\geq 5 \mathrm{~mm}, \geq 10 \mathrm{~mm}$, or $\geq 15 \mathrm{~mm}$ ) or used commercially available assays (QFT-Gold, QFT-Gold-In-Tube (QIAGEN, Netherlands) or T-SPOT.TB (Oxford Immunotec, Oxford, UK) and reported positive results using the manufacturers' recommended cut points. Studies also had to have a mean or median follow-up of at least 12 months and include at least 10 untreated participants who tested positive for latent tuberculosis infection. We included studies of HIV uninfected general populations and populations at increased risk of tuberculosis, using a broad definition of increased risk (see supplementary S1 for populations selected a priori for inclusion, and methods for classification). We excluded studies that investigated only TST converters, TST boosters, Bacille Calmette-Guérin (BCG) or other experimental vaccinated populations, or participants who previously tested positive. To avoid confounding the interpretation of risk estimates, we further excluded studies of people living with HIV in countries with a tuberculosis incidence of more than 100 cases per 100000 people owing to the high likelihood of reinfection, ${ }^{19}$ as well as studies conducted in countries with a general population prevalence of HIV of more than 5\% where authors did not explicitly exclude, or report separately, people living with HIV. In our primary analysis, to minimise selection and publication bias, we further limited included groups to those where at least 100 participants were tested and untreated.

\section{Data extraction and quality assessment}

Standardised data extraction forms were developed a priori (see fields in supplementary table S4). Two reviewers (JRC and NW) independently extracted and quality assessed data, with discrepancies resolved through consensus. Extracted information included study design; location; duration and method of followup; diagnostic test used; distribution of population sex, age, and other information we believed might be relevant (eg, antiretroviral use); diagnostic test results and treatment information for latent tuberculosis infection; and the number of active tuberculosis events and method used for diagnosis. If mean follow-up duration was not reported among participants with a positive test result, we used the study mean or median follow-up time. If population size was not reported, we back calculated this value using reported rates, 
follow-up time, and number of cases. We allowed 1 $\mathrm{mm}$ deviations from TST cut points for inclusion in the respective categories (eg, $\geq 6 \mathrm{~mm}$ grouped with $\geq 5 \mathrm{~mm}$ ) and allowed one year deviations from age categories (eg, <19 years grouped with $<18$ years). Of 17 authors contacted for additional information, eight responded. Quality assessment was based on nine items from a modified quality assessment of diagnostic accuracy studies (QUADAS-2) tool ${ }^{20}$ (see criteria in supplementary table S5). Studies meeting $\geq 7$ criteria, 4-6 criteria, or $\leq 3$ criteria were classified as high, moderate, and low quality, respectively.

\section{Outcomes}

The primary outcome was the incidence of tuberculosis per 1000 person years, and a secondary outcome was the cumulative incidence of tuberculosis among untreated participants who tested positive for latent tuberculosis infection. An additional secondary outcome was the incidence rate ratio of tuberculosis among participants who tested positive for latent infection compared with those who tested negative (ie, indeterminates were excluded) in studies reporting both groups.

\section{Data analysis}

In the planned primary analysis, for each identified group and tuberculosis test we estimated the pooled primary and secondary outcomes using random effects meta-analysis. We pooled the results for QFT-Gold and QFT-Gold-In-Tube (referred to as QFT). ${ }^{21}$ We analysed outcomes separately for TST indurations at $5 \mathrm{~mm}, 10$ $\mathrm{mm}$, and $15 \mathrm{~mm}$ cut points, T-SPOT.TB, QFT, and both IGRAs pooled together.

In planned secondary analysis, for studies reporting results of TST and IGRA, we calculated the incidence rate ratio among people with concordant (same test results) and discordant (test results differ) results. In further planned secondary analyses, we included all studies, regardless of the number of participants tested, and conducted a priori defined stratified analysis based on method of follow-up (passive $v$ active), median year of study start $(\leq 2005$ $v \geq 2006$ ), study design (randomised controlled trial $v$ retrospective cohort $v$ prospective cohort), mean or median duration of follow-up ( $\leq 24$ months $v>24$ months), annual tuberculosis incidence in country of study (<30 per $100000 v \geq 30$ per 100000), country level income (high $v$ upper middle, lower middle, and low), method of tuberculosis diagnosis (microbiological $v$ microbiological and clinical $v$ not specified), and study quality (high $v$ moderate $v$ low). In post hoc analysis, we conducted stratified analysis based on proportion of patients excluded because of treatment for latent tuberculosis infection $(<20 \% v$ $\geq 20 \%$ ) and stratified analysis based on country level policy of BCG vaccination (multiple or post-infancy $v$ vaccination only at birth or none) according to the BCG World Atlas. $^{22}$

All analyses were performed with package meta (version 4.9-5) in R. Individual study outcome estimates were log or logit transformed and random effects meta-analysed using generalised linear mixed models with a Poisson distribution; pooled estimates were back transformed. This method has the advantage of avoiding zero cell corrections that could bias estimates when events are rare. ${ }^{23}{ }^{24}$ Heterogeneity was assessed using the $\mathrm{I}^{2}$ statistic. ${ }^{25}$ Publication bias was not assessed statistically, as factors other than results, such as investigator motivation and funding, might influence why a study is published, commonly used tests do not always agree, few population groups analysed met the minimum criteria for statistical assessment of publication bias, and lack of statistical significance or funnel plot asymmetry does not indicate lack of publication bias. ${ }^{26-30}$

\section{Patient and public involvement}

Patients were not involved in the development of the research question or its outcome measures, conduct of the research, or preparation of the manuscript.

\section{Results}

Overall, 5166 unique citations were identified. After screening of titles and abstracts, 505 remained for full text assessment, of which 122 studies were included. Of these, 102 studies reported results with TST, 71 with IGRA, and 51 with both (see supplementary figure 1). Among the 102 studies using TST, 70 (69\%) explicitly stated the TST method used; of these, 69 (99\%) used the Mantoux method and one (1\%) used the Heaf method while reporting Mantoux equivalent measurements. Additionally, 78 (76\%) studies reported the type and dose of tuberculin used; of these, $69(88 \%)$ used two tuberculin units of RT23 or five tuberculin units of purified protein derivative. According to quality assessment, the most commonly detected sources of bias in studies were follow-up $(n=89 ; 73 \%)$ and selection $(n=72 ; 59 \%)$; only eight (7\%) studies had no detected source of bias, and 47 (39\%) studies were graded as low quality. In 52 studies (43\%), at least $20 \%$ of participants were excluded because of treatment for latent tuberculosis infection. The median year of study start was 2006, 79 (65\%) studies were prospective cohort studies, 87 (71\%) took place in high income countries, and 104 (85\%) took place in countries with an annual tuberculosis incidence of less than 100 per 100000 population (see supplementary table S6).

Three studies of 33811 people with a TST induration of $\geq 10 \mathrm{~mm}$ from HIV uninfected general population samples were included, and 120 studies evaluated 19 different at risk populations, comprising 269 distinct cohorts with 116197 people. Across these studies, 32507 people (48 studies, 61 cohorts), 49482 people (59 studies, 71 cohorts), and 14477 people (15 studies, 20 cohorts) had a positive TST result at cut points of 5 $\mathrm{mm}, 10 \mathrm{~mm}$, and $15 \mathrm{~mm}$, respectively, whereas 10811 people (44 studies, 55 cohorts) and 2564 people (18 studies, 21 cohorts) had a positive result for QFT-Gold or QFT-Gold-In-Tube and T-SPOT.TB, respectively. In total, 6356 people (20 studies, 41 cohorts) were tested 
using both a TST and an IGRA and had concordant positive or discordant positive results. Supplementary tables S7 to S9 report individual study characteristics and outcomes.

\section{Primary analysis}

In thethreestudiesamong HIV uninfected samplesfrom the general population, the incidence of tuberculosis among 33811 participants with a TST induration of $\geq 10 \mathrm{~mm}$ was 0.3 (95\% confidence interval 0.1 to 1.1 ) per 1000 person years; these studies did not report on rates among those with TST $<10 \mathrm{~mm}$ (table 1). For the primary analysis, at risk populations included 37 cohorts of active tuberculosis contacts (18 cohorts using TST and 19 cohorts using IGRAs), 18 cohorts of people living with HIV (nine using TST and nine using IGRAs), 40 cohorts of populations with other immune deficiency (22 with TST and 18 with IGRAs), and 41 cohorts of populations with characteristics affecting exposure and immunocompetency (27 using TST and 14 using IGRAs).

Rick of tuberculosis among exposed populations Among all contacts-both close (ie, people with regular, extensive contact with a person with tuberculosis) and casual (ie, people with regular, but much less frequent contact with a person with tuberculosis) with a TST $\geq 5 \mathrm{~mm}$, the incidence of tuberculosis was 8.4 (95\% confidence interval 5.6 to 12.6$)$ per 1000 person years, whereas among all contacts with a positive IGRA result, the tuberculosis incidence rate was 17.0 (12.9 to 22.4) per 1000 person years (table 1 ; supplementary figure 2 ). These rates are 28 times and 56 times higher, respectively, than the incidence in the three general population studies (table 1). Incidence rates after a positive TST result were not substantially higher in studies defining a positive TST result as $\geq 10 \mathrm{~mm}$ (9.4 per 1000 person years). Overall, contacts with a TST result of $\geq 5 \mathrm{~mm}$ experienced tuberculosis at 6.0 -fold (95\% confidence interval 3.9-fold to 9.2-fold) the rate of contacts with a TST result of $<5 \mathrm{~mm}$, whereas the corresponding incidence rate ratio was 10.8 (95\% confidence interval 6.1 to 19.0) among contacts with a positive IGRA result compared with contacts with a negative IGRA result (table 1; supplementary figure 3). Most pooled incidence rate estimates were highly heterogeneous, but this was not appreciably reduced through stratified analyses (see supplementary tables S10 to S19).

\section{Risk of tuberculosis among people living with HIV} The risk of tuberculosis among people living with HIV was substantially greater than rates among the general population (table 2). Among people living with HIV, the pooled incidence rates in those with a TST result of $\geq 5 \mathrm{~mm}$ were higher than in those with a positive IGRA result ( $27.1 v 16.9$ per 1000 person years; table 2; supplementary figure 4). Over a mean follow-up of 2.9 years, the cumulative incidence of tuberculosis among people living with HIV was $7.1 \%$ (95\% confidence interval $4.2 \%$ to $11.9 \%$ )

\begin{tabular}{|c|c|c|c|c|c|c|c|c|c|}
\hline \multirow[b]{2}{*}{$\begin{array}{l}\text { Population and definition } \\
\text { of positive test result }\end{array}$} & \multicolumn{6}{|c|}{ Among test positive participants } & \multicolumn{3}{|c|}{ Compared with test negative participants } \\
\hline & $\begin{array}{l}\text { No of } \\
\text { cohorts }\end{array}$ & $\begin{array}{l}\text { No of } \\
\text { participants }\end{array}$ & $\begin{array}{l}\text { Total (mean) person } \\
\text { years follow-up }\end{array}$ & $\begin{array}{l}\text { Active TB } \\
\text { events (\%)* }\end{array}$ & $\begin{array}{l}\text { TB rate per } 1000 \text { person } \\
\text { years }(95 \% \mathrm{Cl}) \dagger\end{array}$ & $I^{2}(\%)$ & $\begin{array}{l}\text { No of } \\
\text { cohorts }\end{array}$ & $\begin{array}{l}\text { Incidence rate } \\
\text { ratio }(95 \% \mathrm{Cl}) \dagger\end{array}$ & $I^{2}(\%)$ \\
\hline \multicolumn{10}{|l|}{ General population‡ } \\
\hline $\mathrm{TST} \geq 10 \mathrm{~mm}$ & 3 & 33811 & $249093(7.4)$ & $55(0.2)$ & $0.3(0.1$ to 1.1$)$ & 96 & - & - & - \\
\hline \multicolumn{10}{|c|}{ Close and casual contacts (together)§ } \\
\hline \multicolumn{10}{|c|}{ All age groups: } \\
\hline All IGRA positive & 9 & 2199 & $6667(3.0)$ & $89(4.0)$ & $13.3(10.8$ to 16.4$)$ & 0 & 7 & $8.4(5.2$ to 13.5$)$ & 19 \\
\hline $\mathrm{TST} \geq 5 \mathrm{~mm}$ & 4 & 7861 & $32708(4.2)$ & $227(2.8)$ & $8.4(4.3$ to 16.5$)$ & 95 & 4 & $12.5(6.9$ to 22.5$)$ & 48 \\
\hline TST $\geq 10 \mathrm{~mm}$ & 5 & 5728 & $22561(3.9)$ & $97(2.6)$ & $9.4(4.0$ to 21.8$)$ & 93 & 5 & $6.9(3.3$ to 14.4$)$ & 54 \\
\hline \multicolumn{10}{|l|}{ Age $<18$ years: } \\
\hline TST $\geq 5 \mathrm{~mm}$ & 2 & 1169 & $2766(2.4)$ & $55(6.6)$ & $14.7(1.4$ to 153.6$)$ & 97 & 2 & $20.1(2.9$ to 140.6$)$ & 77 \\
\hline $\mathrm{TST} \geq 10 \mathrm{~mm}$ & 4 & 1002 & $2357(2.4)$ & $69(7.2)$ & 23.4 (8.0 to 68.4$)$ & 92 & 3 & $21.2(6.4$ to 70.2$)$ & 75 \\
\hline \multicolumn{10}{|l|}{ Close contacts onlyq } \\
\hline \multicolumn{10}{|l|}{ All age groups: } \\
\hline All IGRA positive & 7 & 1663 & $4617(2.8)$ & $114(6.5)$ & 22.4 (12.1 to 41.8$)$ & 87 & 6 & 13.1 (3.3 to 51.9) & 86 \\
\hline $\mathrm{TST} \geq 5 \mathrm{~mm}$ & 9 & 7626 & $37696(4.9)$ & $340(3.8)$ & $10.5(7.7$ to 14.3$)$ & 83 & 8 & 3.7 (2.1 to 6.5$)$ & 66 \\
\hline $\mathrm{TST} \geq 10 \mathrm{~mm}$ & 13 & 8849 & $29970(3.4)$ & $296(2.7)$ & $10.3(6.1$ to 17.4$)$ & 94 & 11 & $2.5(1.3$ to 4.8$)$ & 86 \\
\hline \multicolumn{10}{|l|}{ Age $<18$ years: } \\
\hline QFT-GIT positive & 2 & 159 & 305 (1.9) & $6(2.3)$ & $14.5(1.7$ to 120.5$)$ & 27 & 1 & $13(4.9$ to 34.6$)$ & $\mathrm{N} / \mathrm{A}$ \\
\hline TST $\geq 10 \mathrm{~mm}$ & 3 & 584 & $1152(2.0)$ & $17(2.4)$ & $12.6(2.3$ to 69.8$)$ & 88 & 2 & $4.2(1.8$ to 9.7$)$ & 0 \\
\hline \multicolumn{10}{|c|}{ All studies in contacts above pooled** } \\
\hline All IGRA positive & 19 & 4060 & $11743(2.9)$ & $211(4.6)$ & $17.0(12.9$ to 22.4$)$ & 81 & 15 & $10.8(6.1$ to 19.0$)$ & 74 \\
\hline $\mathrm{TST} \geq 5 \mathrm{~mm}$ & 18 & 19628 & $90266(4.6)$ & $615(2.8)$ & $8.4(5.6$ to 12.6$)$ & 96 & 16 & 6.0 (3.9 to 9.2$)$ & 70 \\
\hline TST $\geq 10 \mathrm{~mm}$ & 27 & 18414 & $69596(3.8)$ & $461(2.6)$ & $9.4(6.3$ to 14.1$)$ & 94 & 22 & $4.1(2.6$ to 6.4$)$ & 82 \\
\hline \multicolumn{10}{|c|}{$\begin{array}{l}\text { TST=tuberculin skin test; IGRA=interferon-gamma release assay; QFT-GIT=QuantiFERON Gold-In-Tube; N/A=not applicable. } \\
\text { *Percentage represents pooled cumulative incidence estimated from random effects meta-analysis. } \\
\text { †Pooled estimates using random-effects meta-analysis. } \\
\text { fData provided for reference. Populations are from British Columbia and Saskatchewan, Canada, and Florida, USA. } \\
\text { §Studies were conducted in populations of contacts, with no stratification by intensity of exposure or closeness of contact. } \\
\text { IStudies were conducted in populations of contacts that were either solely close contacts or had outcomes reported stratified by close and casual contact. } \\
\text { ** Representative of all studies in contacts, regardless of intensity of exposure. All studies included if }>100 \text { participants were tested. Participants are not double counted (i.e. we did not pool } \\
\text { cohorts coming from the same study for which participants may fall into more than one group, such as the all ages group and the <18 age group, instead we only included the all age group). }\end{array}$} \\
\hline
\end{tabular}




\begin{tabular}{|c|c|c|c|c|c|c|c|c|c|}
\hline \multirow[b]{2}{*}{$\begin{array}{l}\text { Population and definition } \\
\text { of positive test result }\end{array}$} & \multicolumn{6}{|c|}{ Among test positive participants } & \multicolumn{3}{|c|}{ Compared with test negative participants } \\
\hline & $\begin{array}{l}\text { No of } \\
\text { cohorts }\end{array}$ & $\begin{array}{l}\text { No of } \\
\text { participants }\end{array}$ & $\begin{array}{l}\text { Total (mean) person } \\
\text { years follow-up }\end{array}$ & $\begin{array}{l}\text { Active TB } \\
\text { events }(\%)^{\star}\end{array}$ & $\begin{array}{l}\text { TB rate per } 1000 \text { person } \\
\text { years }(95 \% \mathrm{Cl}) \dagger\end{array}$ & $\mathrm{I}^{2}(\%)$ & $\begin{array}{l}\text { No of } \\
\text { cohorts }\end{array}$ & $\begin{array}{l}\text { Incidence rate } \\
\text { ratio }(95 \% \mathrm{Cl})\end{array}$ & $\mathrm{I}^{2}(\%)$ \\
\hline \multicolumn{10}{|l|}{ General population‡: } \\
\hline TST $\geq 10 \mathrm{~mm}$ & 3 & 33811 & $249093(7.4)$ & $55(0.2)$ & $0.3(0.1$ to 1.1$)$ & 96 & - & - & - \\
\hline \multicolumn{10}{|l|}{ Dialysis: } \\
\hline All IGRA positive & 4 & 375 & $871(2.3)$ & $3(0.8)$ & $3.4(1.1$ to 10.7$)$ & 0 & 1 & $7.0(0.7$ to 67$)$ & $\mathrm{N} / \mathrm{A}$ \\
\hline $\mathrm{TST} \geq 5 \mathrm{~mm}$ & 2 & 151 & $379(2.5)$ & $10(2.0)$ & $11.8(0.7$ to 200.7$)$ & 59 & 1 & $1.5(0.7$ to 3.3$)$ & N/A \\
\hline TST $\geq 10 \mathrm{~mm}$ & 3 & 129 & $253(2.0)$ & $19(14.7)$ & $100.6(41.6$ to 243.2$)$ & 71 & 3 & $2.6(1.4$ to 4.8$)$ & 0 \\
\hline \multicolumn{10}{|l|}{ HIV: } \\
\hline All IGRA positive & 9 & 573 & $1646(2.9)$ & $28(5.1)$ & $16.9(10.5$ to 27.3$)$ & 29 & 7 & $11.0(4.6$ to 26.2$)$ & 45 \\
\hline $\mathrm{TST} \geq 5 \mathrm{~mm}$ & 9 & 1285 & $3782(2.9)$ & $148(7.1)$ & 27.1 (15.0 to 49.0$)$ & 87 & 7 & 11.1 (6.2 to 19.9) & 46 \\
\hline \multicolumn{10}{|l|}{ Silicosis: } \\
\hline $\mathrm{TST} \geq 10 \mathrm{~mm}$ & 2 & 352 & $1465(4.2)$ & $54(12.5)$ & 36.9 (28.2 to 48.1$)$ & 0 & 1 & $1.7(0.5$ to 5.5$)$ & N/A \\
\hline \multicolumn{10}{|l|}{ Transplant§: } \\
\hline All IGRA positive: & 9 & 481 & $993(2.1)$ & $8(1.6)$ & $6.7(2.2$ to 20.4$)$ & 33 & 6 & $2.5(1.0$ to 6.0$)$ & 0 \\
\hline $\mathrm{TST} \geq 5 \mathrm{~mm}$ & 7 & 518 & $1716(3.3)$ & $8(0.1)$ & $1.0(0$ to 64$)$ & 64 & 7 & 0.6 (0.1 to 3.0$)$ & 30 \\
\hline TST $\geq 10 \mathrm{~mm}$ & 3 & 105 & $215(2.0)$ & $5(0.6)$ & 3.7 (0 to 861.5) & 88 & 3 & $2.4(0.9$ to 6.4$)$ & 0 \\
\hline \multicolumn{10}{|l|}{ Immunosuppressants§: } \\
\hline All IGRA positive & 3 & 110 & $248(2.3)$ & $4(0.6)$ & $4.8(0.1$ to 279.2$)$ & 73 & 1 & 48.1 (5.4 to 430.7$)$ & $\mathrm{N} / \mathrm{A}$ \\
\hline TST $\geq 5 \mathrm{~mm}$ & 5 & 212 & $551(2.6)$ & $3(1.4)$ & 5.4 (1.8 to 16.9$)$ & 0 & 3 & 6.6 (1.8 to 24.2$)$ & 0 \\
\hline \multicolumn{10}{|l|}{ Diabetes§: } \\
\hline All IGRA positive & 2 & 334 & $842(2.5)$ & $4(1.2)$ & $4.4(0.9$ to 22.0$)$ & 8 & 1 & Not calculable & N/A \\
\hline \multicolumn{10}{|c|}{$\begin{array}{l}\text { TST=tuberculin skin test; IGRA=interferon-gamma release assay; N/A=not applicable. } \\
\text { *Percentage represents pooled cumulative incidence estimated from random effects meta-analysis. } \\
\text { †Pooled estimates using random effects meta-analysis. } \\
\text { ‡Data provided for reference. Populations are from British Columbia and Saskatchewan, Canada, and Florida, USA. } \\
\S \text { See supplementary S1 for a further description of studies comprising these analyses. }\end{array}$} \\
\hline
\end{tabular}

among those with a positive TST result of $\geq 5 \mathrm{~mm}$ and $5.1 \%(3.5 \%$ to $7.2 \%)$ among those with a positive IGRA result. After stratifying by year of study start, tuberculosis incidence rates among people living with HIV with a TST result of $\geq 5 \mathrm{~mm}$ were 40.2 (95\% confidence interval 24.9 to 64.7) per 1000 person years in six studies started before 2006 and 9.5 (2.1 to 42.5$)$ per 1000 person years in three studies started from 2006 onwards. Compared with people living with HIV and corresponding negative test results for latent tuberculosis infection, those with a TST result of $\geq 5 \mathrm{~mm}$, or a positive IGRA result, experienced tuberculosis incidence rates that were 11-fold higher (see supplementary figure 5).

\section{Risk of tuberculosis among populations with conditions affecting immunity other than HIV}

The risk of tuberculosis among populations with other conditions affecting immunity were more variable but still consistently greater than rates among the general population (table 2). The incidence rate for tuberculosis was high among people with silicosis and a TST result of $\geq 10 \mathrm{~mm}$, at 36.9 (95\% confidence interval 28.2 to 48.1) per 1000 person years. Among people requiring dialysis, tuberculosis incidence rates were 100.6 (41.6 to 243.2 ) per 1000 person years among those with a TST result of $\geq 10 \mathrm{~mm}$ and 3.4 (1.1 to 10.7) per 1000 person years among those who with a positive IGRA result. All tuberculosis among people requiring dialysis occurred in studies where less than $20 \%$ of the study population was excluded because of treatment for latent tuberculosis infection (see supplementary table S18). Among transplant recipients, the tuberculosis rate was 3.7 ( 0 to 861.5 ) per 1000 person years among those with a TST result of $\geq 10 \mathrm{~mm}$ and
6.7 (2.2 to 20.4) per 1000 person years among those with a positive IGRA result. Among people receiving immunosuppressant drugs, the risk of tuberculosis was similar among those with a TST result of $\geq 5 \mathrm{~mm}$ or a positive IGRA result (5.4 and 4.8 per 1000 person years, respectively). In two studies that evaluated the risk of tuberculosis among people with diabetes and a positive IGRA result the rate was 4.4 (95\% confidence interval 0.9 to 22.0) per 1000 person years. Incidence rate ratios between people with a positive TST result and a negative TST result ranged from 0.6 to 2.6 for those who required dialysis, received a transplant, or had silicosis; the incidence rate ratio was 7.0 in the one study reporting IGRA results in people requiring dialysis.

\section{Risk of tuberculosis among populations with characteristics affecting exposure and immunocompetency}

The risk of tuberculosis was variable among populations with characteristics that affect exposure to tuberculosis and immunocompetency (table 3). Among participants older than 65 years, despite increased tuberculosis rates (3.6 and 11.2 per 1000 person years for a TST result of $\geq 10 \mathrm{~mm}$ and positive IGRA result, respectively), incidence rate ratios were low (2.2 and 1.3, respectively). Among prisoners, the risk of tuberculosis and the corresponding incidence rate ratio in those with a TST result of $\geq 10 \mathrm{~mm}$ was high (45.0 per 1000 person years and 31.0, respectively). Among recent immigrants or refugees, most from countries with a tuberculosis incidence greater than 30 per 100000 (see supplementary S1), a TST of $\geq 5 \mathrm{~mm}$ or a positive IGRA result were associated with similar tuberculosis rates (9.5 and 10.7 per 1000 person 
years, respectively) and cumulative incidences (3.5\% and $3.1 \%$, respectively), although incidence estimates with TST were highly heterogeneous $\left(\mathrm{I}^{2}=90 \%\right)$. Among immigrants or refugees, the paradoxical finding of decreasing tuberculosis rates as cut points for positive TST results increased was due to high rates among studies initiated before 2006 (see supplementary table S15). When considering only studies initiated from 2006 onwards, immigrants or refugees with a TST result of $\geq 10 \mathrm{~mm}$ or positive IGRA result had similar incidence rates of tuberculosis (7.4 and 9.8 per 1000 person years, respectively)-among those with a TST result of $\geq 5 \mathrm{~mm}$, this rate was 5.4 (95\% confidence interval 3.5 to 8.3 ) per 1000 person years. Studies of participants with occupational risk factors were largely among healthcare workers in whom risk was low, regardless of test used.

\section{Summary}

To aid decisions to test for or treat latent tuberculosis infection, or both, we plotted tuberculosis incidence rates and corresponding incidence rate ratios for all at risk populations included in the primary analysis (fig 1). Populations in the upper right quadrant have both high incidence rates and high incidence rate ratios, whereas populations in the lower left quadrant have both low incidence rates and low incidence rate ratios. People living with HIV, tuberculosis contacts, prisoners, and recent immigrants or refugees had the most favourable incidence rate to incidence rate ratio relation. Supplementary table S20 further summarises these results.

\section{Secondary analysis}

In stratified, secondary analyses the method and duration of follow-up, country level incidence of tuberculosis, and study quality seemed to influence the incidence rate of tuberculosis across numerous populations (see supplementary tables S10 to S13). The design of the study, time when the study was conducted, country level income, method of tuberculosis diagnosis, proportion of people excluded who received treatment for latent tuberculosis infection, and country policy on BCG vaccination did not seem to have consistent effects on tuberculosis incidence rates (see supplementary tables S14 to S19).

Risk of tuberculosis with concordant and discordant TST and IGRA results could be estimated using six studies among contacts that reported results from concurrent dual testing. The incidence rate ratio for tuberculosis was 19.1 (95\% confidence interval 2.9 to 127.3) among participants with positive results for both tests compared with those with negative results for both tests (table 4). Pooled tuberculosis rates per 1000 person years were high in those with dual positive test results (range 30.6-43.1), compared with estimated rates in those with discordant positive test results (6.2-16.5) and in those with dual negative test results (2.6-3.1). In all the other at risk populations, few studies reported results of concurrent dual testing (see supplementary table S21 for results).
When the primary analysis was repeated and all studies regardless of the size of the included population were included, the results and their interpretation did not appreciably change. Supplementary tables 22-24 show these analyses and outcomes for populations not included in the primary analysis.

\section{Discussion}

This review provides detailed information on the risk of tuberculosis associated with positive or negative results from commonly used tests for latent tuberculosis infection, in people with a wide variety of underlying conditions or in epidemiological risk groups. Findings of this review underscore the benefits of testing for latent infection, given the high incidence rate ratio of positive test results compared with negative test results in many at risk groups, and the potential benefits of treating people in many at risk subgroups with a positive test result for latent tuberculosis infection. Regardless of test used, high rates of tuberculosis were seen among people living with HIV, tuberculosis contacts, prisoners, and people with silicosis, whereas modest rates were seen among recent immigrants or refugees, people requiring dialysis, transplant recipients, and people requiring immunosuppressant drugs. This information should help guide the clinical decisions to test, perform a second test, and treat latent tuberculosis infection in the context of programme priorities and local assessments of feasibility, acceptability, and cost effectiveness. $^{3} 15$

We found a considerable difference in the incidence rate of tuberculosis-but not the incidence rate ratio, which remained consistent-among people living with HIV with a TST result of $\geq 5 \mathrm{~mm}$ in studies conducted before 2006 compared with those conducted from 2006 onwards (40.2 v 9.5 per 1000 person years). This difference could be attributable to healthcare system strengthening ${ }^{31}$ or differences in the study populations included in each period. This difference might also reflect the expanded use of antiretroviral treatment, which increased from a range of $0-61 \%$ in pre-2006 studies to $40-71 \%$ in studies initiated from 2006 onwards. $^{32}$ Among people requiring dialysis or undergoing transplantation, observed rates of disease were variable; estimates for incidence rate ratios were, however, consistently low. We speculate this might have reflected low sensitivity of all tests in these populations with severe immunodeficiency, ${ }^{33}$ or selective treatment by knowledgeable providers.

For several populations-notably people with diabetes, receiving immunosuppressant drugs, or with a body mass index of less than $18.5 \mathrm{~kg} / \mathrm{m}^{2}$-few studies reported the incidence of tuberculosis after a positive test result for latent tuberculosis infection among untreated participants. Future research among these populations to better define the risk of tuberculosis should be prioritised. This is best accomplished by making use of high quality, administrative databases, which should provide the opportunity to control for several possible confounders. 


\begin{tabular}{|c|c|c|c|c|c|c|c|c|c|}
\hline \multirow[b]{2}{*}{$\begin{array}{l}\text { Population and definition } \\
\text { of positive test result }\end{array}$} & \multicolumn{6}{|c|}{ Among test positive participants } & \multicolumn{3}{|c|}{ Compared with test negative participants } \\
\hline & $\begin{array}{l}\text { No of } \\
\text { cohorts }\end{array}$ & $\begin{array}{l}\text { No of } \\
\text { participants }\end{array}$ & $\begin{array}{l}\text { Total (mean) person } \\
\text { years follow-up }\end{array}$ & $\begin{array}{l}\text { Active TB } \\
\text { events }(\%)^{*}\end{array}$ & $\begin{array}{l}\text { TB rate per } 1000 \text { person } \\
\text { years }(95 \% \mathrm{Cl}) \dagger\end{array}$ & $I^{2}(\%)$ & $\begin{array}{l}\text { No of } \\
\text { cohorts }\end{array}$ & $\begin{array}{l}\text { Incidence rate ratio } \\
(95 \% \mathrm{Cl}) t\end{array}$ & $\mathrm{I}^{2}(\%)$ \\
\hline \multicolumn{10}{|l|}{ General populationf: } \\
\hline TST $\geq 10 \mathrm{~mm}$ & 3 & 33811 & $249093(7.4)$ & $55(0.2)$ & $0.3(0.1$ to 1.1$)$ & 96 & - & - & - \\
\hline \multicolumn{10}{|l|}{ Age $<18$ years: } \\
\hline TST $\geq 10 \mathrm{~mm}$ & 3 & 3388 & $30642(9.0)$ & $26(0.5)$ & $0.8(0.1$ to 6.4$)$ & 93 & 1 & $4.9(2.7$ to 8.8$)$ & $\mathrm{N} / \mathrm{A}$ \\
\hline \multicolumn{10}{|l|}{ Age $\geq 65$ years: } \\
\hline QFT-G(IT) positive & 2 & 1879 & $3752.3(2.0)$ & $42(2.2)$ & $11.2(8.3$ to 15.1$)$ & 0 & 1 & $1.3(0.1$ to 14.1$)$ & $\mathrm{N} / \mathrm{A}$ \\
\hline TST $\geq 10 \mathrm{~mm}$ & 2 & 1376 & $3937.5(2.9)$ & $14(1.0)$ & $3.6(2.1$ to 6.0$)$ & 0 & 2 & $2.2(0.9$ to 5.2$)$ & 0 \\
\hline \multicolumn{10}{|c|}{ Recent immigrant or refugee arrival§: } \\
\hline All IGRA positive & 4 & 1597 & $4673(2.9)$ & $50(3.1)$ & $10.7(8.1$ to 14.1$)$ & 0 & 4 & 10.9 (6.3 to 18.9$)$ & 0 \\
\hline TST $\geq 5 \mathrm{~mm}$ & 3 & 2145 & $8132(3.8)$ & $103(3.5)$ & $9.5(4.3$ to 21.0$)$ & 90 & 2 & $6.4(2.4$ to 16.5$)$ & 0 \\
\hline $\mathrm{TST} \geq 10 \mathrm{~mm}$ & 3 & 10735 & $103142(9.6)$ & $133(1.7)$ & $2.7(1.0$ to 7.1$)$ & 96 & 2 & $4.0(2.1$ to 7.9$)$ & 34 \\
\hline \multicolumn{10}{|l|}{ Prisoners: } \\
\hline TST $\geq 10 \mathrm{~mm}$ & 3 & 739 & $777(1.1)$ & $21(6.1)$ & $45.0(9.0$ to 224.6$)$ & 93 & 3 & $31.0(4.1$ to 233.9$)$ & 0 \\
\hline \multicolumn{10}{|l|}{ Occupational risk factors: } \\
\hline All IGRA positive & 8 & 1467 & $3665(2.5)$ & $11(0.7)$ & $3.0(1.7$ to 5.4$)$ & 0 & 3 & $4.9(0.5$ to 48.3$)$ & 50 \\
\hline $\mathrm{TST} \geq 5 \mathrm{~mm}$ & 3 & 401 & $837(2.1)$ & $3(0.7)$ & 3.6 (1.2 to 11.1$)$ & 0 & 1 & 0.5 (0.1 to 5.0$)$ & $\mathrm{N} / \mathrm{A}$ \\
\hline TST $\geq 10 \mathrm{~mm}$ & 6 & 2696 & $5580(2.1)$ & $14(0.7)$ & $2.8(1.3$ to 6.1$)$ & 34 & 4 & 1.7 (0.7 to 4.2) & 0 \\
\hline
\end{tabular}

\section{Comparison with other studies}

Previous meta-analyses have estimated the risk of tuberculosis in at risk populations compared with populations without that characteristic-for example, risk in people with diabetes versus without diabetes. ${ }^{34}$ These previous estimates have not managed to distinguish risk for progression to active tuberculosis from the risk of being infected. By providing estimates of tuberculosis risk among those with a positive test result for latent tuberculosis infection, this metaanalysis more clearly and directly informs the clinical question encountered by providers: what is the likelihood that active disease will develop in someone with a positive test result for latent tuberculosis infection? Furthermore, this meta-analysis enables estimation of tuberculosis risk among those with a

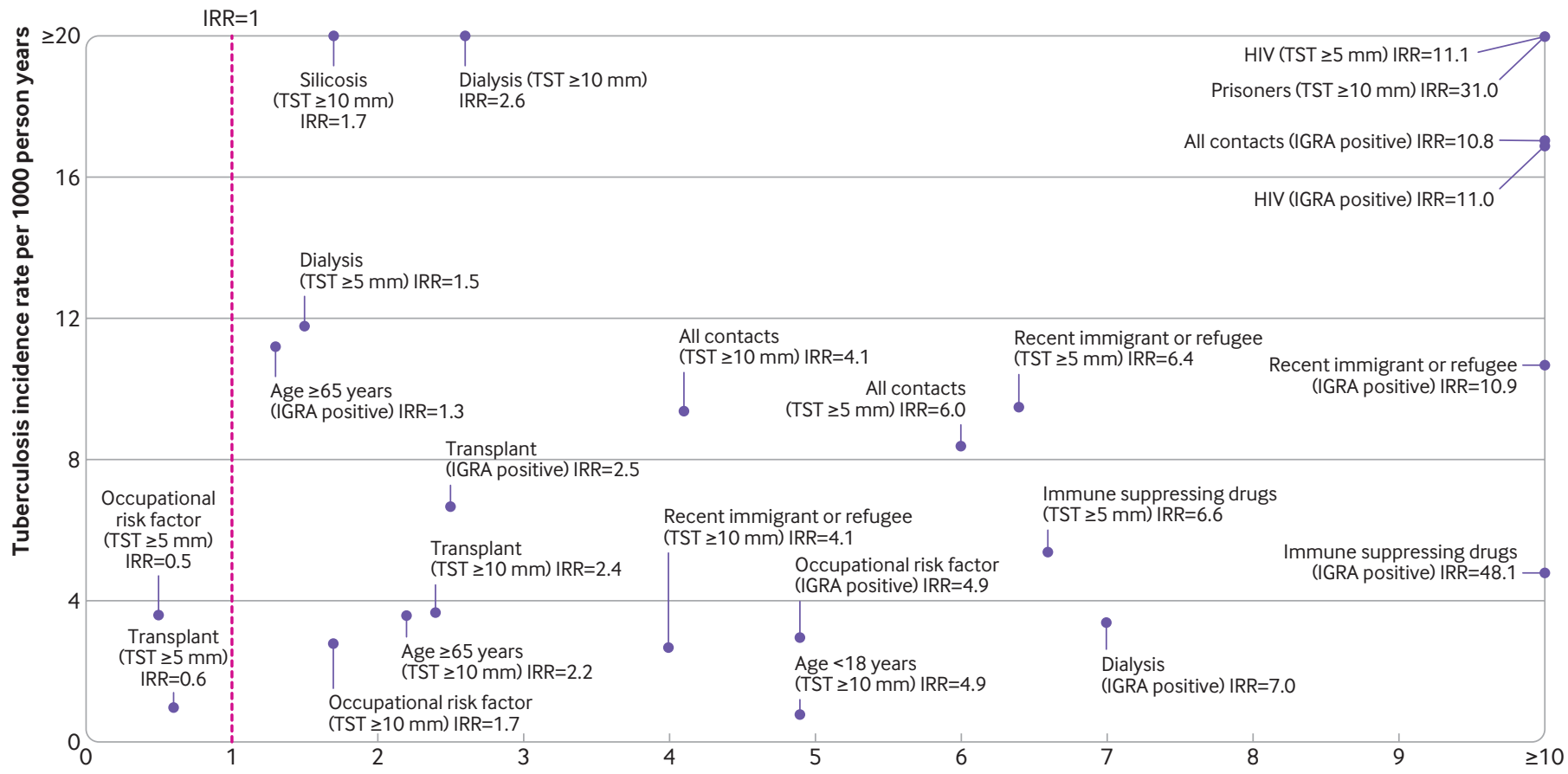

Tuberculosis incidence rate ratio

Fig 1 | Summary results of primary analysis showing risk of developing tuberculosis per 1000 person years versus the incidence rate ratio (IRR). The results for tuberculin skin test (TST) are presented for cut points of $\geq 5 \mathrm{~mm}$ and $\geq 10 \mathrm{~mm}$ for indurations, and the results for interferon-gamma release assay (IGRA) are presented as all types combined. Included populations have a minimum of two studies in the meta-analysis of incidence rate and have a calculable estimate for the incidence rate ratio 


\begin{tabular}{|c|c|c|c|c|c|c|c|}
\hline Group comparison & $\begin{array}{l}\text { No of } \\
\text { studies }\end{array}$ & $\begin{array}{l}\text { No of } \\
\text { participants }\end{array}$ & $\begin{array}{l}\text { Total (mean) person } \\
\text { years follow-up }\end{array}$ & $\begin{array}{l}\text { Active TB events } \\
(\%)^{\star}\end{array}$ & $\begin{array}{l}\text { TB rate per } 1000 \text { per- } \\
\text { son years }(95 \% \mathrm{Cl}) \dagger\end{array}$ & $\begin{array}{l}\text { Incidence rate } \\
\text { ratio }(95 \% \mathrm{Cl}) \dagger\end{array}$ & $\mathrm{I}^{2}(\%)$ \\
\hline General population, TST positiveł & 3 & 33811 & $249093(7.4)$ & $55(0.2)$ & $0.3(0.1$ to 1.1$)$ & - & - \\
\hline \multicolumn{8}{|l|}{ Dual positive $v$ dual negative } \\
\hline $\begin{array}{l}\text { IGRA positive and TST positive } v \text { IGRA } \\
\text { negative and TST negative } \S\end{array}$ & 4 & $588 v 2724$ & $1576(2.7) \vee 5856(2.1)$ & $36(8.7) \vee 15(0.6)$ & $\begin{array}{l}30.6(10.9 \text { to } 85.9) v \\
2.6(1.2 \text { to } 5.6)\end{array}$ & 19.1 (2.9 to 127.3$)$ & 80 \\
\hline \multicolumn{8}{|c|}{ Dual positive v discordant (positive/negative) } \\
\hline $\begin{array}{l}\text { IGRA positive and TST positive } v \text { IGRA } \\
\text { positive and TST negative }\end{array}$ & 3 & $566 \vee 476$ & $1464(2.6) \vee 956(2.0)$ & $34(8.8)$ v $29(3.3)$ & $\begin{array}{l}37.3(10.5 \text { to } 132.4) v \\
16.5(3.7 \text { to } 74.8)\end{array}$ & $3.0(0.2$ to 40.7$)$ & 83 \\
\hline $\begin{array}{l}\text { IGRA positive and TST positive } v \text { IGRA } \\
\text { negative and TST positive**}\end{array}$ & 6 & $641 \vee 1428$ & $1715(2.7)$ v $4410(3.1)$ & $47(12.1)$ v 29 (1.8) & $\begin{array}{l}43.1(21.1 \text { to } 88.0) v \\
6.2(2.4 \text { to } 15.9)\end{array}$ & $7.6(1.6$ to 36.7$)$ & 86 \\
\hline \multicolumn{8}{|c|}{ Discordant (positive/negative) v dual negative } \\
\hline $\begin{array}{l}\text { IGRA positive and TST negative } v \text { IGRA } \\
\text { negative and TST negativet† }\end{array}$ & 3 & $476 \vee 2643$ & $956(2.0) \vee 5541(2.1)$ & $29(3.3) \vee 15(0.7)$ & $\begin{array}{l}16.5(3.7 \text { to } 74.8) v \\
3.1(1.4 \text { to } 6.6)\end{array}$ & $5.1(2.4$ to 10.8$)$ & 0 \\
\hline $\begin{array}{l}\text { IGRA negative and TST positive } v \text { IGRA } \\
\text { negative and TST negative } \neq \neq\end{array}$ & 4 & $672 \vee 2724$ & $1534(2.3) \vee 5856(2.1)$ & $18(2.4) \vee 15(0.6)$ & $\begin{array}{l}9.1(3.0 \text { to } 28.0) v \\
2.6(1.2 \text { to } 5.6)\end{array}$ & 3.6 (1.8 to 7.2$)$ & 0 \\
\hline
\end{tabular}

TST=tuberculin skin test; IGRA=interferon-gamma release assay; N/A=not applicable.

*Percentage represents pooled cumulative incidence estimated from random effects meta-analysis.

tPooled estimates using random effects meta-analysis.

‡Data provided for reference. Populations are from British Columbia and Saskatchewan, Canada, and Florida, USA.

§One study used the T-SPOT.TB IGRA, while the remaining three used the QFT-GIT (QuantiFERON Gold-In-Tube) IGRA. One study, using QFT-GIT, was exclusively in contacts aged <18 years.

IOne study used the T-SPOT.TB IGRA, whereas the remaining two used the QFT-GIT IGRA. One study, using QFT-GIT, was exclusively in contacts aged <18 years.

${ }^{* *}$ One study used the T-SPOT.TB IGRA, whereas the remaining five used the QFT-GIT IGRA. Two studies, using QFT-GIT, were exclusively in contacts aged $<18$ years

t†One study used the T-SPOT.TB IGRA, whereas the remaining two used the QFT-GIT IGRA. One study, using QFT-GIT, was exclusively in contacts aged <18 years.

$\neq \neq$ One study used the T-SPOT.TB IGRA, whereas the remaining three used the QFT-GIT IGRA. One study, using QFT-GIT, was exclusively in contacts aged <18 years.

negative test result for latent infection by dividing the incidence rate among those with a positive test result by the corresponding incidence rate ratio. These estimates of risk should be useful in the shared decision making process between providers and patients in the treatment of latent tuberculosis infection.

A consistent finding for both IGRA and TST was that for many populations the incidence rate ratio for people with a positive result versus negative result was high. This was particularly noticeable among people living with HIV. In resource limited settings, the World Health Organization recommends the treatment of latent tuberculosis infection for people living with HIV even if testing for latent infection is not available, although noting that such testing is preferred. ${ }^{10}$ For both TST and IGRA we found large, identical incidence rate ratios among people living with HIV. This strongly suggests that in areas with a high incidence of tuberculosis, improved access to the TST, an inexpensive test requiring little infrastructure to implement, could provide important benefits to people living with HIV, by focusing limited resources on treating those most at risk. Furthermore, the benefits of treating latent tuberculosis infection have been consistently shown to be greater among people living with HIV with a positive TST result compared with those with a negative result. ${ }^{35} 36$ However, we recognise that the absolute risk of tuberculosis among people living with HIV with a negative TST result is still higher than that in the general population, and this population might still benefit from treatment for latent tuberculosis infection. ${ }^{37}$

In our secondary analysis of incidence rate ratios among contacts being tested with both a TST and an IGRA, we found that among this high risk population, incidence rate ratios were high for both concordant positive and discordant positive groups, when compared with the concordant negative group. This implies that in the presence of a single negative test result for latent tuberculosis infection in a high risk person, there might be value in performing a second test. Whether to use TST or IGRA first is, however, unclear, as the direct comparison of these tests was not an objective of this meta-analysis. This question was assessed recently, but with inconsistent results. ${ }^{38}$

\section{Strengths and limitations of this review}

Our meta-analysis has key strengths. In our literature search we maximised sensitivity, and to further enhance sensitivity we applied loose criteria for citations to qualify for full text review. Using generalised linear mixed models to perform random effects meta-analysis reduced biases that would be introduced through other methods of pooling, such as inverse variance, ${ }^{2324}$ that require zero cell corrections. Finally, we estimated several effect measures that policymakers and providers might use when determining whether to test, treat, or test and treat people for latent tuberculosis infection.

This meta-analysis has some limitations. Many studies used passive follow-up for detection of active tuberculosis and were of low quality; factors which we found to be associated with lower observed rates of active tuberculosis. Some studies included people who previously had tuberculosis. In such studies, however, these participants were most often a minority of the included population, and tuberculosis rates did not differ between studies with and without people who previously had tuberculosis. Publication bias was not statistically assessed but cannot be excluded. The included studies in this review were published from 1990 to 2019. The risk of tuberculosis over this period might be variable owing to changes in health systems and population health, among others, resulting in higher tuberculosis rates being observed in studies conducted earlier in this period. However, we did 
not identify a consistent difference in tuberculosis risk between studies conducted before and studies conducted after 2006. In studies using a TST, rounding of induration measurements might introduce bias, but since this is a common issue in clinical practice, we do not think it affects the generalisability of our results. Most included studies were conducted in settings with a tuberculosis incidence of less than 100 per 100000 population, potentially restricting generalisability of our results to settings with a low incidence of tuberculosis and low risk of reinfection.

Many national and international guidelines ${ }^{2}$ 8-11 recommend treatment of latent tuberculosis infection for many of the populations included in this metaanalysis. Of the studies included, about $40 \%$ prescribed treatment for latent tuberculosis infection to $20 \%$ or more of participants. It might be expected that in these studies those untreated were at lowest risk of disease, resulting in an underestimation of the population risk of tuberculosis. Given the aggregate nature of the data it is difficult to control for this possible confounding factor and determine the potential effect it had on estimates. In stratified analyses comparing studies prescribing treatment for latent tuberculosis infection to less than $20 \%$ of the study population compared with $20 \%$ or more we did not notice a consistent trend that studies prescribing treatment to $20 \%$ or more of the population had lower rates of tuberculosis.

Other factors could contribute to underestimation of tuberculosis risk, such as possible dropouts, death, or emigration. Additionally, among non-HIV infected immunocompromised populations, such as people requiring dialysis, immortal time bias might be present as tuberculosis incidence is highest in the immediate months after diagnosis. ${ }^{39} 40$

Alternatively, some factors might result in overestimation of risk. It is possible some populations, such as tuberculosis contacts, included people with additional characteristics, such as diabetes, that further increased their risk of tuberculosis. The mean follow-up time in most studies was two to three years, so our estimates might overestimate long term risk, after many years. However, our estimates of cumulative incidence help clarify total future risk of tuberculosis in a fixed timeframe; the high estimates of risk seen underscore the importance of treatment for latent tuberculosis infection to reduce that risk. Lastly, although $85 \%$ of studies were conducted in countries with an annual tuberculosis incidence of less than 100 per 100000 , re-exposure and reinfection could have contributed to incident tuberculosis.

\section{Conclusion and policy implications}

Among members of certain risk groups with a positive TST or IGRA result, incidence rates for tuberculosis were high compared with members of the same group with a negative test result for latent tuberculosis infection, or people in the general population with a positive TST result but no other risk factor. The highest rates were seen among tuberculosis contacts, people living with HIV, recent immigrants or refugees, people requiring dialysis, people with silicosis, and prisoners. The findings of this review should encourage providers to test for latent tuberculosis infection and consider treatment in people with one of the risk factors associated with an increased risk of incident tuberculosis.

We thank Olivia Oxlade and James Johnston for critical review of a draft of this manuscript and Andrea Benedetti for advice on statistical analysis.

Contributors: JRC and DM had full access to all the data in the study and take responsibility for the integrity of the data and the accuracy of the data analysis. They are the guarantors. All authors conceived and designed the study; acquired, analysed, or interpreted the data; critically revised the manuscript; and provided administrative, technical, or material support. JRC drafted the manuscript and carried out the statistical analysis. JRC and DM obtained the funding. DM supervised the study. The corresponding author attests that all listed authors meet authorship criteria and that no others meeting the criteria have been omitted.

Funding: JRC is supported by a postdoctoral fellowship from the Fonds de Recherche du Québec Santé (award \#258907) and NW is supported through a Canadian Institutes of Health Research grant (FRD331745). The funders had no role in the design and conduct of the study; collection, management, analysis, and interpretation of the data; preparation, review, or approval of the manuscript; and decision to submit the manuscript for publication.

Competing interests: All authors have completed the ICMJE uniform disclosure and declare: JRC is supported by a postdoctoral fellowship from the Fonds de Recherche du Québec Santé (award \#258907) and NW is supported through a Canadian Institutes of Health Research grant (FRD331745); no financial relationships with any organisations that might have an interest in the submitted work in the previous three years, no other relationships or activities that could appear to have influenced the submitted work.

Ethical approval: Not required.

Data sharing: No additional data available.

The lead authors (JRC and DM) affirm that the manuscript is an honest, accurate, and transparent account of the study being reported; that no important aspects of the study have been omitted; and that any discrepancies from the study as planned have been explained.

Dissemination to participants and related patient and public communities: The results of the meta-analysis will be disseminated to patients, providers, policymakers, and others through various mediums, including social media, press releases, and academic and institutional networks. The results of this meta-analysis will be used to inform the next iteration of the Canadian Tuberculosis Standards and revision of an online tuberculosis risk calculator, both of which are accessible online, without restriction. The tuberculin skin test interpreter is available at https://www.tstin3d.com/.

This is an Open Access article distributed in accordance with the Creative Commons Attribution Non Commercial (CC BY-NC 4.0) license, which permits others to distribute, remix, adapt, build upon this work non-commercially, and license their derivative works on different terms, provided the original work is properly cited and the use is non-commercial. See: http://creativecommons.org/licenses/ by-nc/4.0/.

1 Dye C, Glaziou P, Floyd K, Raviglione M. Prospects for tuberculosis elimination. Annu Rev Public Health 2013;34:271-86. doi:10.1146/ annurev-publhealth-031912-114431

2 American Thoracic Society. Targeted tuberculin testing and treatment of latent tuberculosis infection. MMWR Recomm Rep 2000;49(RR6):1-51

3 Muñoz L, Stagg HR, Abubakar I. Diagnosis and Management of Latent Tuberculosis Infection. Cold Spring Harb Perspect Med 2015;5:5. doi:10.1101/cshperspect.a017830

4 Menzies D, Pai M, Comstock G. Meta-analysis: new tests for the diagnosis of latent tuberculosis infection: areas of uncertainty and recommendations for research. Ann Intern Med 2007;146:340-54. doi:10.7326/0003-4819-146-5-200703060-00006

5 Campbell JR, Dowdy D, Schwartzman K. Treatment of latent infection to achieve tuberculosis elimination in low-incidence countries. PLOS Med 2019:16:e1002824 doi:10.1371/journal.pmed.1002824

6 Andrade RJ, Tulkens PM. Hepatic safety of antibiotics used in primary care. J Antimicrob Chemother 2011;66:1431-46. doi:10.1093/jac/ dkr159 
7 Kiazyk S, Ball TB. Latent tuberculosis infection: An overview. Can Commun Dis Rep 2017;43:62-6. doi:10.14745/ccdr.v43i34a01

8 Australian Government Department of Health. CDNA National Guidelines for Public Health Units - Management of TB. Australian Government Department of Health, 2015.

9 Public Health Agency of Canada. Canadian Tuberculosis Standards. 7th ed. Government of Canada, 2014

10 WHO. Latent tuberculosis infection: Updated and consolidated guidelines for programmatic management. World Health Organization, 2018

11 National Institute for Health and Care Excellence. Tuberculosis: Guidance and guidelines. National Institute for Health and Care Excellence, 2016.

12 Horsburgh CRJr. Priorities for the treatment of latent tuberculosis infection in the United States. N Engl / Med 2004;350:2060-7. doi:10.1056/NEJMsa031667

13 Getahun H, Matteelli A, Chaisson RE, Raviglione M. Latent Mycobacterium tuberculosis infection. N Engl J Med 2015;372:212735. doi:10.1056/NEJMra1405427

14 van Leth F, van der Werf MJ, Borgdorff MW. Prevalence of tuberculous infection and incidence of tuberculosis: a re-assessment of the Styblo rule. Bull World Health Organ 2008;86:20-6. doi:10.2471/ BLT.06.037804

15 Dobler CC, Bosnic-Anticevich S, Armour CL. Physicians' perspectives on communication and decision making in clinical encounters for treatment of latent tuberculosis infection. ER/ Open Res 2018:4:4. doi:10.1183/23120541.00146-2017

16 Liberati A, Altman DG, Tetzlaff J, et al. The PRISMA statement for reporting systematic reviews and meta-analyses of studies that evaluate healthcare interventions: explanation and elaboration. BMJ 2009;339:b2700. doi:10.1136/bmj.b2700

17 Stroup DF, Berlin JA, Morton SC, et al. Meta-analysis of observational studies in epidemiology: a proposal for reporting. Meta-analysis Of Observational Studies in Epidemiology (MOOSE) group. JAMA 2000;283:2008-12. doi:10.1001/jama.283.15.2008

18 Horsburgh CRJr, O’Donnell M, Chamblee S, et al. Revisiting rates of reactivation tuberculosis: a population-based approach. Am J Respir Crit Care Med 2010;182:420-5. doi:10.1164/rccm.200909$13550 \mathrm{C}$

19 Chaisson RE, Churchyard GJ. Recurrent tuberculosis: relapse, reinfection, and HIV. J Infect Dis 2010;201:653-5. doi:10.1086/650531

20 Whiting PF, Rutjes AWS, Westwood ME, et al, QUADAS-2 Group. QUADAS-2: a revised tool for the quality assessment of diagnostic accuracy studies. Ann Intern Med 2011;155:529-36. doi:10.7326/0003-4819-155-8-201110180-00009

21 Mahomed H, Hughes EJ, Hawkridge T, et al. Comparison of mantoux skin test with three generations of a whole blood IFN-gamma assay for tuberculosis infection. Int I Tuberc Lung Dis 2006:10:310-6.

22 Zwerling A, Behr MA, Verma A, Brewer TF, Menzies D, Pai M. The BCG World Atlas: a database of global BCG vaccination policies and practices. PLoS Med 2011;8:e1001012. doi:10.1371/journal. pmed.1001012

23 Hamza TH, van Houwelingen HC, Stijnen T. The binomial distribution of meta-analysis was preferred to model within-study variability. J Clin Epidemiol 2008;61:41-51. doi:10.1016/j. jclinepi.2007.03.016

24 Spittal MJ, Pirkis J, Gurrin LC. Meta-analysis of incidence rate data in the presence of zero events. BMC Med Res Methodol 2015;15:42. doi:10.1186/s12874-015-0031-0

25 Higgins JPT, Thompson SG, Deeks JJ, Altman DG. Measuring inconsistency in meta-analyses. BMJ 2003;327:557-60. doi:10.1136/bmj.327.7414.557
26 Macaskill P, Walter SD, Irwig L. A comparison of methods to detect publication bias in meta-analysis. Stat Med 2001;20:641-54. doi:10.1002/sim.698.

27 Higgins JPT, Green S. Cochrane Handbook for Systematic Reviews of Interventions. The Cochrane Collaboration 2011. https:// handbook-5-1.cochrane.org/ (accessed 9 Dec 2019).

28 Guyatt GH, Oxman AD, Montori V, et al. GRADE guidelines: 5. Rating the quality of evidence--publication bias. / Clin Epidemiol 2011;64:1277-82. doi:10.1016/i.jclinepi.2011.01.011

29 Mueller M, D’Addario M, Egger M, et al. Methods to systematically review and meta-analyse observational studies: a systematic scoping review of recommendations. BMC Med Res Methodol 2018;18:44. doi:10.1186/s12874-018-0495-9

30 Sterne JAC, Sutton AJ, loannidis JPA, et al. Recommendations for examining and interpreting funnel plot asymmetry in meta-analyses of randomised controlled trials. BMJ 2011;343:d4002. doi:10.1136/ bmj.d4002

31 Atun R, Weil DEC, Eang MT, Mwakyusa D. Health-system strengthening and tuberculosis control. Lancet 2010;375:2169-78. doi:10.1016/S0140-6736(10)60493-X

32 Tiruneh G, Getahun A, Adeba E. Assessing the Impact of Isoniazid Preventive Therapy (IPT) on Tuberculosis Incidence and Predictors of Tuberculosis among Adult Patients Enrolled on ART in Nekemte Town, Western Ethiopia: A Retrospective Cohort Study. Interdiscip Perspect Infect Dis 2019;2019:1413427. doi:10.1155/2019/1413427

33 Pai M, Denkinger CM, Kik SV, et al. Gamma interferon release assays for detection of Mycobacterium tuberculosis infection. Clin Microbiol Rev 2014;27:3-20. doi:10.1128/CMR.00034-13

34 Jeon CY, Murray MB. Diabetes mellitus increases the risk of active tuberculosis: a systematic review of 13 observational studies. PLoS Med 2008;5:e152. doi:10.1371/journal.pmed.0050152

35 Briggs MA, Emerson C, Modi S, Taylor NK, Date A. Use of isoniazid preventive therapy for tuberculosis prophylaxis among people living with HIV/AIDS: a review of the literature. J Acquir Immune Defic Syndr 2015;68(Suppl 3):S297-305. doi:10.1097/ QAl.0000000000000497

36 Wilkinson D, Squire SB, Garner P. Effect of preventive treatment for tuberculosis in adults infected with HIV: systematic review of randomised placebo controlled trials. BMJ 1998;317:625-9. doi:10.1136/bmj.317.7159.625

37 Slovis BS, Plitman JD, Haas DW. The case against anergy testing as a routine adjunct to tuberculin skin testing. JAMA 2000;283:2003-7. doi:10.1001/jama.283.15.2003

38 Auguste P, Tsertsvadze A, Pink J, et al. Comparing interferongamma release assays with tuberculin skin test for identifying latent tuberculosis infection that progresses to active tuberculosis: systematic review and meta-analysis. BMC Infect Dis 2017;17:200. doi:10.1186/s12879-017-2301-4

39 Campbell JR, Johnston JC, Ronald LA, et al. Screening for Latent Tuberculosis Infection in Migrants With CKD: A Cost-effectiveness Analysis. Am J Kidney Dis 2019;73:39-50. doi:10.1053/j. ajkd.2018.07.014

40 Chia S, Karim M, Elwood RK, FitzGerald JM. Risk of tuberculosis in dialysis patients: a population-based study. Int J Tuberc Lung Dis 1998;2:989-91.

Supplementary information: Details on the systematic review and data extraction; individual study characteristics and outcomes; and additional analyses, figures, and explanations in support of the study results 